Gefässchirurgie 2022 $27: 170-175$

https://doi.org/10.1007/s00772-022-00873-6

Angenommen: 24. Januar 2022

Online publiziert: 4. März 2022

๑) Der/die Autor(en) 2022

\title{
Präklinische Daten zum Trauma in Extremitätenarterien
}

\author{
Beeinflussen Entfernung des Unfallorts und \\ Transportmodalität das Ergebnis?
}

\author{
Josef Klocker · Sophia Anna Elisabeth Heuberger · Alexandra Gratl · \\ Michaela Kluckner · Sabine Wipper \\ Universitätsklinik für Gefäßchirurgie, Medizinische Universität Innsbruck, Innsbruck, Österreich
}

\section{Zusammenfassung}

Hintergrund: Die Universitätsklinik Innsbruck (Österreich) ist ein Traumazentrum mit großem Einzugsgebiet. Wir analysierten retrospektiv Aspekte der präklinischen Versorgung der PatientInnen mit traumatischen Extremitätenarterienverletzungen (Entfernung des Unfallorts, boden- vs. luftgebundener Transport, direkte vs. indirekte Einlieferung) und deren möglichen Einfluss auf Ergebnisparameter (Letalität, Extremitätenerhalt, Folgeoperationen und Länge des Krankenhausaufenthalts). Material und Methoden: Retrospektive Kohortenstudie unter Einschluss aller PatientInnen mit rekonstruierten Extremitätenarterienverletzungen, die in der Zeit vom 01.01.2004 bis zum 31.12.2018 behandelt worden waren. Ausschlusskriterien waren iatrogene Gefäßverletzungen, konservativ behandelte Läsionen, mittels Ligatur oder Embolisation behandelte Läsionen und Läsionen an Seitenästen der Hauptarterien. Ergebnisse: Insgesamt wurden 113 PatientInnen eingeschlossen, davon 59 an der oberen und 54 an der unteren Extremität. Unfallmechanismen waren 34,5\% Sport- und Freizeitunfälle, $23 \%$ Arbeitsunfälle und 20,4\% Verkehrsunfälle. Der PatientInnentransport erfolgte signifikant häufiger direkt als indirekt $(59,3 \%$ vs. 40,7\%; $p<0,05)$, allerdings mit beträchtlichen Unterschieden an oberer bzw. unterer Extremität abhängig vom Unfallmechanismus. Sowohl der direkte als auch indirekte Transport erfolgte signifikant häufiger luftgebunden via Hubschrauber. Entfernung des Unfallorts, Transportmodalität und direkte bzw. indirekte Zutransferierung hatten keinen signifikanten Einfluss auf Letalität, Extremitätenerhalt, Häufigkeit von Folgeoperationen und die Länge des Krankenhausaufenthalts.

Schlussfolgerung: Unsere retrospektive Datenanalyse zeigt, dass die Zentralisierung der Versorgung von traumatischen Extremitätenarterienverletzungen keine Nachteile für PatientInnen, aus entfernteren Landesteilen nach sich zieht. Ebenso gibt es keinen Unterschied der Ergebnisse hinsichtlich der direkten Einlieferung oder späteren Zutransferierung nach zügig durchgeführter Erstversorgung und Erstuntersuchung auswärts.

\section{Schlüsselwörter}

Gefäßverletzung · Extremität · Präklinik · Patiententransport · Klinisches Ergebnis

Die englische Version dieses Beitrags ist unter https://doi.org/10.1007/s00772-022-00872-7 zufinden.

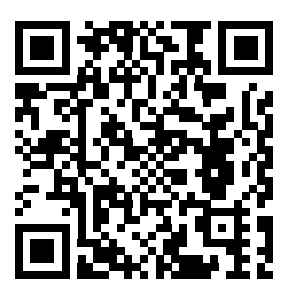

QR-Code scannen \&Beitrag online lesen

\section{Hintergrund}

Das klinische Ergebnis und die Versorgungsqualität von traumatisch bedingten Gefäßverletzungen kann anhand typischer Endpunkte gemessen und bewertet werden. Für Arterienverletzungen an den Extremitäten sind dies:
1. Die Anzahl der verletzungsbedingten Todesfälle (Letalität) - diese wird in der Fachliteratur uneinheitlich berichtet, entweder als intraoperative, perioperative, 30-Tages- oder In-HospitalLetalität. Diese berücksichtigen jedoch nicht diejenigen Traumen, die vor Einlieferung ins Krankenhaus bzw. vor 
Einleitung der spezifischen Behandlung im Krankenhaus zum Tod geführt haben.

2. Der Extremitätenerhalt. Er beinhaltet allerdings in der gefäßchirurgisch publizierten Fachliteratur nicht Traumen (mit oder ohne Gefäßbeteiligung), die entsprechend dem Prinzip „life before limb" ohne Rekonstruktionsversuch einer primären Amputation zugeführt wurden.

3. Die Anzahl der Reinterventionen bzw. Folgeoperationen - vaskulär oder nicht vaskulär bedingt - sowohl im frühen postoperativen als auch im Langzeitverlauf nach Jahren. Dazu sind einerseits Revisionen der arteriellen Rekonstruktion und andererseits Folgeoperationen im Sinne einer Weichteilrekonstruktion bzw. nach Fasziotomie oder zur Behandlung von knöchernen und/oder nervalen Begleitverletzungen zu rechnen.

4. Das funktionelle Ergebnis im Langzeitverlauf. Die Problematik einer langfristig eingeschränkten Extremitätenfunktion unterscheidet sich zwischen oberer und unterer Extremität, und beeinflusst gravierend die Lebensqualität. Dies kann mithilfe spezifischer Scores gemessen und quantifiziert werden und ist vor allem in der Gutachtermedizin bedeutsam, wenn es um eine Minderung der Erwerbsfähigkeit geht.

Die Gefäßchirurgie Innsbruck ist für traumatisch bedingte Extremitätenarterienverletzungen seit Jahrzehnten de facto das einzige Versorgungszentrum für das gesamte Bundesland Tirol (mit etwa 760.000 Einwohnern bzw. einer Fläche von ca. $12.650 \mathrm{~km}^{2}$ ). Teilweise werden auch Patientlnnen aus benachbarten Regionen und in relevantem Umfang auch Touristen, die in der Region Tirol verunfallen, behandelt. In Anbetracht des großen und vielfach nur erschwert erreichbaren Einzugsgebiets stellt sich natürlich die Frage, ob einzelne Bedingungen der präklinischen Versorgung die Ergebnisse beeinflussen, z.B. die Entfernung des Unfallorts, die Modalität des Patiententransports (boden- vs. luftgebunden) oder die Tatsache, ob direkt oder indirekt (nach Erstabklärung bzw. Voruntersuchungen in einem anderen Krankenhaus) zutransferiert wurde. Es stellt sich die Frage, ob die Auswertung der etablierten Ergebnisparameter für einzelne Patientengruppen Nachteile zeigen, also ein singuläres Versorgungszentrum problematisch ist.

Die vorliegende Datenerhebung wurde von Frau Sophia Anna Elisabeth Heuberger als Diplomarbeit an der Universitätsklinik für Gefäßchirurgie durchgeführt und unter dem Titel „Präklinische Daten zur Versorgung der traumatischen Extremitätenarterienverletzungen" an der Medizinischen Universität Innsbruck eingereicht und angenommen.

\section{Fragestellungen}

Retrospektiv wurde untersucht

- wie weit entfernt der Unfallort vom Versorgungszentrum Innsbruck war, wobei dies sowohl für den bodengebundenen (Straßenkilometer) als auch den luftgebundenen (Annahme: direkte Luftlinie) Transport kalkuliert wurde;

- wie die Zutransferierung tatsächlich erfolgte (bodengebunden vs. luftgebunden);

- ob die Zutransferierung direkt ins Versorgungszentrum oder indirekt also nach Erst- bzw. Voruntersuchung in einer Krankenanstalt der Regel- und Grundversorgung erfolgte;

- ob die genannten Kenndaten (Entfernung des Unfallorts bzw. Modus der Zutransferierung) zu Unterschieden in den klinischen Ergebnissen führten, gemessen anhand typischer Endpunkte (Letalität, Extremitätenerhalt, vaskuläre und nichtvaskuläre Folgeoperationen, Länge des Krankenhausaufenthalts).

Nicht untersucht wurde, ob und inwieweit die Umsetzung der etablierten Richtlinien zur Versorgung von Schwerverletzten (z. B. S3-Leitlinie Polytrauma der Deutschen Gesellschaft für Unfallchirurgie) im Einzelfall vor Ort erfolgte, ob die Gefäßverletzung überhaupt präklinisch erkannt wurde und wer warum die Entscheidung über die Transportmodalität (bodengebunden vs. luftgebunden sowie direkt vs. indirekt) getroffen hat.

\section{Studiendesign und Untersu- chungsmethoden}

Alle PatientInnen, die im Zeitraum zwischen dem 01.01.2004 und dem 31.12.2018 an der Universitätsklinik für Gefäßchirurgie in Innsbruck aufgrund einer traumatisch bedingten Verletzung einer Hauptarterie der oberen oder unteren Extremität operativ oder endovaskulär behandelt wurden, sind retrospektiv identifiziert und eingeschlossen worden. Der Zeitraum ab 01.01.2004 wurde deshalb gewählt, weil - beginnend mit diesem Jahr - die Patientendokumentation vollständig EDVgestützt vorliegt, sowohl externe Notarztprotokolle und Transferierungsberichte als auch sämtliche Daten am Standort.

Ausschlusskriterien waren: iatrogen verursachte Extremitätenarterienverletzungen, konservativ behandelte Verletzungen, mittels Ligatur oder Embolisation behandelte Läsionen und Läsionen an Seitenästen der Hauptarterien.

Für alle Patienten wurden retrospektiv aus dem Patientendokumentationssystem Daten zu Unfallmechanismus, Unfallort und Transportmodalität (bodengebunden vs. luftgebunden; direkt oder indirekt) erhoben und ausgewertet. Diese Daten wurden dann hinsichtlich Details der Verletzung (Lokalisation, betroffenes Gefäßsegment, Begleitverletzungen), der operativen bzw. endovaskulären Versorgung sowie des klinischen Ergebnisses der PatientInnen (Mortalität, Extremitätenerhalt, vaskuläre und nichtvaskuläre Folgeoperationen, Länge des Krankenhausaufenthalts) ergänzt.

Die Auswertung der erhobenen Daten erfolgte mittels deskriptiver Statistik. Der Gruppenvergleich der Zielvariablen erfolgte für die kategorischen Parameter mittels Chi-Quadrat-Test, für quantitative Parameter mittels Mann-Whitney-U-Test. Eine Signifikanz im Gruppenvergleich wurde für $p<0,05$ angegeben.

\section{Ergebnisse}

Insgesamt wurden im 15-jährigen Untersuchungszeitraum an der Universitätsklinik für Gefäßchirurgie in Innsbruck 113 PatientInnen (davon $77 \%$ männlich) mit Extremitätenarterienverletzungen behandelt, 59 an der oberen und 54 an der 
Tab. 1 Patientencharakteristika - Unfallmechanismen

\begin{tabular}{|l|l|l|l|}
\hline & Obere Extremität & Untere Extremität & Gesamt \\
\hline Anzahl PatientInnen $(n)$ & 59 & 54 & 113 \\
\hline Sport- u. Freizeitunfall $n(\%)$ & $19(32,2)$ & $20(37,0)$ & $39(34,5)$ \\
\hline Verkehrsunfall $n(\%)$ & $8(13,6)$ & $15(27,8)$ & $23(20,4)$ \\
\hline Arbeitsunfall $n(\%)$ & $14(23,7)$ & $12(22,2)$ & $26(23,0)$ \\
\hline Nicht dokumentiert $n(\%)$ & $18(30,5)$ & $7(13,0)$ & $25(22,1)$ \\
\hline
\end{tabular}

Tab. 2 Transportmodalität: Zutransferierung direkt vs. indirekt bzw. boden-vs. luftgebunden

\begin{tabular}{|l|l|l|l|}
\hline & Obere Extremität & Untere Extremität & Gesamt \\
\hline Anzahl Patienten $(n)$ & 59 & 54 & 113 \\
\hline Zutransferierung direkt $n(\%)$ & $37(62,7)$ & $30(55,6)$ & $67(59,3)$ \\
\hline Direkt luftgebunden (\%) & 64,7 & 61,5 & 63,3 \\
\hline Direkt bodengebunden (\%) & 35,3 & 38,5 & 36,7 \\
\hline Luft- vs. bodengebunden & $p<0,05$ & $p<0,05$ & $p<0,05$ \\
\hline Zutransferierung indirekt $n(\%)$ & $22(37,3)$ & $24(44,4)$ & $46(40,7)$ \\
\hline Indirekt luftgebunden (\%) & 63,6 & 71,4 & 68 \\
\hline Indirekt bodengebunden (\%) & 36,4 & 28,6 & 32 \\
\hline Luft- vs. bodengebunden & $n .5$. & $p<0,05$ & $p<0,05$ \\
\hline Zutransferierung direkt vs. indirekt & $p<0,05$ & $n . s$. & $p<0,05$ \\
\hline $\begin{array}{l}\text { Im Falle einer indirekten Zutransferierung wurde ausschließlich der Sekundärtransport/ } \\
\text { Überstellungstransport gewertet }\end{array}$ & & \\
\hline
\end{tabular}

Tab. 3 Transportmodalität bei Sport- und Freizeitunfällen mit Extremitätenarterienverletzungen: Zutransferierung direkt vs. indirekt

\begin{tabular}{|l|l|l|l|}
\hline- & Obere Extremität & Untere Extremität & Gesamt \\
\hline Sport- u. Freizeitunfall $(n)$ & 19 & 20 & 39 \\
\hline Zutransferierung direkt $n(\%)$ & $11(57,9)$ & $7(35)$ & $18(46,2)$ \\
\hline Zutransferierung indirekt $n(\%)$ & $8(42,1)$ & $13(65)$ & $21(53,8)$ \\
\hline Direkt vs. indirekt & n.s. & n.s. & n.s. \\
\hline
\end{tabular}

Tab. 4 Transportmodalität bei Verkehrsunfällen mit Extremitätenarterienverletzungen: Zutransferierung direkt vs. indirekt

\begin{tabular}{|l|l|l|l|}
\hline- & Obere Extremität & Untere Extremität & Gesamt \\
\hline Verkehrsunfall $(n)$ & 8 & 15 & 23 \\
\hline Zutransferierung direkt $n(\%)$ & $5(62,5)$ & $12(80)$ & $17(73,9)$ \\
\hline Zutransferierung indirekt $n(\%)$ & $3(37,5)$ & $3(20)$ & $6(26,1)$ \\
\hline Direkt vs. indirekt & n. s. & $p<0,05$ & $p<0,05$ \\
\hline
\end{tabular}

unteren Extremität. Das mediane Alter der PatientInnen lag bei 39,1 Jahren (Spanne: 2,5-78,5) an der oberen bzw. 31,8 Jahren (Spanne: 15,4-69,0) an der unteren Extremität.

Die Unfallmechanismen sind in • Tab. 1 zusammengefasst: Am häufigsten waren Sport- und Freizeitunfälle (jeweils an oberer und unterer Extremität deutlich über $30 \%)$. Verkehrsunfälle waren an der unteren Extremität doppelt so häufig wie an der oberen ( $27,8 \%$ vs. $13,6 \%)$. Arbeitsunfälle lagen jeweils bei etwa $23 \%$ vor. In 25 Fällen $(22,1 \%)$ war die Dokumentation teren Extremität (55,6\% vs. 44,4\%; nicht signifikant) (s. - Tab. 2).

Die Subgruppenvergleiche je nach Unfallmechanismus zeigten: Bei Sport- und Freizeitunfällen wurden arterielle Verletzungen an der oberen Extremität rein numerisch häufiger direkt zutransferiert $(57,9 \%)$, dagegen an der unteren Extremität indirekt (65\%) (s. - Tab. 3; Unterschied nicht signifikant). Verkehrsunfälle mit Extremitätenarterienverletzungen wurden sowohl insgesamt $(73,9 \%)$ als auch an der unteren Extremität (80\%) signifikant häufiger direkt zutransferiert (jeweils $p<0,05$ ). Dies galt jedoch nicht für die obere Extremität (s. - Tab.4). Keine signifikanten Unterschiede zeigten sich bei Arbeitsunfällen: Die Häufigkeiten direkter versus indirekter Zutransferierung waren für die obere $(57,1 \%$ vs. $42,9 \%)$ und untere Extremität (58,3\% vs. $41,7 \%$ ) nahezu identisch und nicht signifikant unterschiedlich (s. - Tab. 5).

Luft- vs. bodengebundener direkter Transport

Falls der Transport der PatientInnen direkt vom Unfallort zum Versorgungszentrum Innsbruck erfolgte, wurde dieser signifikant häufiger luftgebunden per Rettungshubschrauber als bodengebunden durchgeführt $(63,3 \%$ vs. $36,7 \% ; p<0,05)$. Der Unterschied zwischen luftgebundenem und bodengebundenem direktem Transport der PatientInnen war wiederum an der oberen $(64,7 \%$ vs. $35,3 \% ; p<0,05)$ stärker ausgeprägt als an der unteren Extremität $(61,5 \%$ vs. $38,5 \% ; p<0,05)$ (s. - Tab. 2).

\section{Luft- vs. bodengebundener indirekter Transport}

hinsichtlich des Unfallmechanismus nicht eindeutig (s. - Tab. 1).

\section{Zutransferierung direkt vs. indirekt}

Der Transport der PatientInnen vom Unfallort zum Versorgungszentrum Innsbruck erfolgte signifikant häufiger direkt als indirekt (59,3\% vs. 40,7\%; $p<0,05)$. Der Unterschied zwischen direktem und indirektem Transport der PatientInnen war an der oberen Extremität (62,7\% vs. 37,3\%; $p<0,05)$ stärker ausgeprägt als an der un-

Für die 46 PatientInnen, die indirekt (Erstversorgung bzw. Erstuntersuchung in einem anderen Krankenhaus) transportiert wurden, waren die Daten hinsichtlich Modalität des Ersttransports (vom Unfallort in das auswärtige Krankenhaus) inkomplett, sodass eine detaillierte Auswertung nicht erfolgen konnte.

Was die Modalität des Überstellungstransports (vom auswärtigen Krankenhaus in das Versorgungszentrum Innsbruck) betrifft, so war auch dieser signifikant häu- 
Tab. 5 Transportmodalität bei Arbeitsunfällen mit Extremitätenarterienverletzungen:Zutransferierung direkt vs. indirekt

\begin{tabular}{|l|l|l|l|}
\hline- & Obere Extremität & Untere Extremität & Gesamt \\
\hline Arbeitsunfall $(n)$ & 14 & 12 & 26 \\
\hline Zutransferierung direkt $n(\%)$ & $8(57,1)$ & $7(58,3)$ & $15(57,7)$ \\
\hline Zutransferierung indirekt $n(\%)$ & $6(42,9)$ & $5(41,7)$ & $11(42,3)$ \\
\hline Direkt vs. indirekt & n.s. & n. s. & n.s. \\
\hline
\end{tabular}

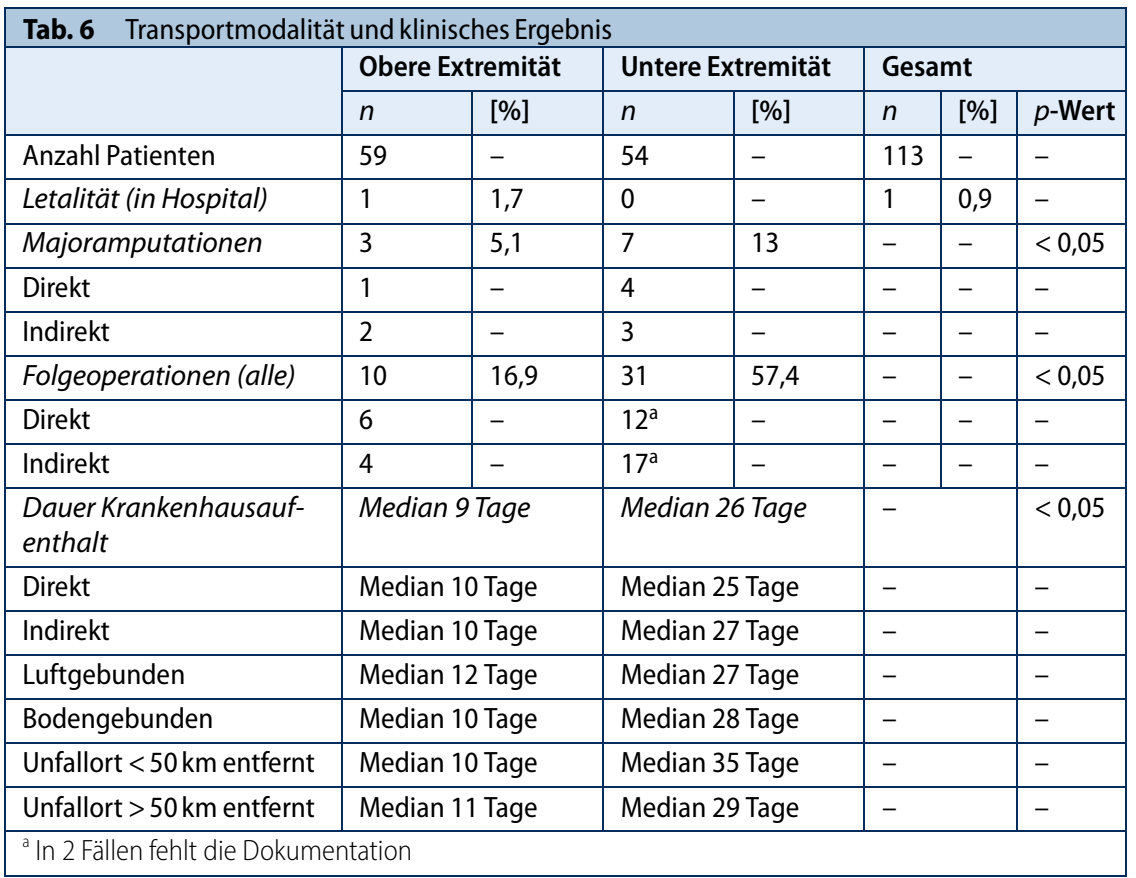

figer luftgebunden als bodengebunden ( $68 \%$ vs. $32 \% ; p<0,05)$. Dieser Unterschied war an der unteren Extremität $(71,4 \%$ vs. $28,6 \% ; p<0,05)$ stärker ausgeprägt als an der oberen $(63,6 \%$ vs. $36,4 \%$; nicht signifikant) (s. - Tab. 2).

Die Subgruppenanalyse für verschiedene Unfallmechanismen wurde aufgrund von geringen Fallzahlen in den Subgruppen nicht berechnet.

\section{Entfernung des Unfallortes}

Der Unfallort lag median luftgebunden $45,3 \mathrm{~km}$ (maximal $96 \mathrm{~km}$ ) und bodengebunden median $71,2 \mathrm{~km}$ (maximal $137,9 \mathrm{~km}$ ) vom Versorgungszentrum Innsbruck entfernt. An der oberen Extremität waren generell die Entfernungen des Unfallortes (luftgebunden: median 53,7 km bzw. maximal $96 \mathrm{~km}$; bodengebunden: median $84,8 \mathrm{~km}$ bzw. maximal $137,9 \mathrm{~km}$ ) signifikant größer als an der unteren Extremität (luftgebunden: median $36,9 \mathrm{~km}$ bzw. maximal $88,1 \mathrm{~km}$; bodengebunden: median $57,6 \mathrm{~km}$ bzw. maximal $108,3 \mathrm{~km}$ ) $(p<0,05)$.

\section{Transportmodalität und klinisches} Ergebnis

Die Krankenhausletalitätlag bei $0,9 \%$ und betraf einen direkt vom Unfallort ins Versorgungszentrum Innsbruck transferierten 60-jährigen Patienten, der nach einem Arbeitsunfall am 4. postoperativen Tag nach Rekonstruktion einer Unterschenkelarterie bei offener Fraktur im kardiogenen Schock verstarb (s. DTab. 6).

Transportmodalität, Entfernung des Unfallorts und direkt oder indirekt erfolgte Einlieferung führten zu keinen signifikanten Unterschieden in den $\mathrm{Am}$ putationsraten (Majoramputationen). Diese lag bei $8,8 \%(n=10)$ und betraf signifikant häufiger die untere Extremität (untere Extremität: $n=7$ bzw. $13 \%$; obere Extremität: $n=3$ bzw. 5,1\%; $p<0,05$ ).

Sowohl Transportmodalität, Entfernung des Unfallorts als auch die direkt oder indirekt erfolgte Einlieferung hatten keinen signifikanten Effekt auf die Häufigkeit von Folgeoperationen jedweder Art (d.h. vaskuläre und nichtvaskuläre Folgeeingriffe). Folgeoperationen betrafen signifikant häufiger die untere Extremität (untere Extremität: $n=31$ bzw. $57,4 \%$; obere Extremität: $n=10$ bzw. 16,9\%; $p<0,05)$. Vaskuläre Folgeoperationen (i.e. Revision wegen postoperativen Verschlüssen der arteriellen Rekonstruktion) erfolgten in 10 Fällen $(18,5 \%)$ an der unteren und 5 -mal $(8,5 \%)$ an der oberen $(p<0,05)$.

Die Entfernung des Unfallorts zum Versorgungszentrum, die Transportmodalität und die direkt oder indirekt erfolgte Einlieferung hatten keinen signifikanten Einfluss auf die mediane Krankenhausaufenthaltsdauer. Auch diese war für die untere Extremität signifikant länger (median: 26 Tage; maximal 88 Tage) als für die obere Extremität (median: 9 Tage; maximal 102 Tage) $(p<0,05)$.

\section{Diskussion}

Unsere Daten zeigen, dass die Behandlungsergebnisse nach Rekonstruktionen von Extremitätenarterienverletzungen im Versorgungszentrum Innsbruck für PatientInnen aus entfernten Landesteilen und mit langen Anfahrtszeiten sowie für PatientInnen nach Erstversorgung und Erstuntersuchung in einem peripheren Krankenhaus (indirekte Zutransferierung) nicht ungünstiger ist als für die anderen Patienten. Auch hatte die Tatsache, ob die Zutransferierung luft- oder bodengebunden erfolgte, keinen signifikanten Einfluss auf das klinische Ergebnis der Kohorte. Dies gilt sowohl für die Letalität, den Extremitätenerhalt, die Häufigkeit von vaskulären und nichtvaskulären Folgeoperationen als auch für die notwendige Krankenhausverweildauer. Die Zentralisierung der Patientenversorgung hat also keinen nachteiligen Effekt für das Kollektiv der PatientInnen, auch nicht für jene die aus entfernten Landesteilen oder indirekt zutransferiert werden.

In der bisher publizierten spezifisch gefäßchirurgisch ausgerichteten Fachliteratur haben wir überraschenderweise keine vergleichbare Auswertung mit ähnlicher Fragestellung vorgefunden. Es gibt 
also aus der Literatur keinen Beleg dafür, dass für PatientInnen mit Extremitätenarterienverletzungen hinsichtlich ihres klinischen Ergebnisses eine bevorzugte Transportmodalität zu wählen oder eine indirekte Zutransferierung in Versorgungszentren nachteilig sei.

Es ist anzumerken, dass in unserer Auswertung die Daten zur Transportmodalität (luftgebunden vs. bodengebunden bzw. direkt vs. indirekt) und zur Entfernung des Unfallorts nicht mit individuell patientenbezogenen Aufzeichnungen der Uhrzeiten in der Versorgungskette (Unfallzeitpunkt, Erstversorgung, Transportbeginn, Transportdauer, Eintreffen im Versorgungszentrum, Operationsbeginn bzw. Zeitpunkt der komplettierten Revaskularisation) gegenkalkuliert werden konnten, da diesbezüglich nur in Einzelfällen eine vollständige Dokumentation vorlag. Somit ist z.B. nicht schlüssig feststellbar, zu welchem "Zeitverlust" der bodengebundene Transport, die indirekte Zutransferierung oder der Transport aus entlegenen Landesteilen geführt hat. Es ist nicht auszuschließen, dass für einzelne PatientInnen unserer Kohorte ein Nachteil aufgrund einer (wodurch auch immer verursachten) Verzögerung entstanden sein könnte. Eine Optimierung der Dokumentation - insbesondere an den Schnittstellen zwischen Prähospitalphase und Übernahme ins Versorgungszentrum - inklusive Erfassung der Zeitachse - ist wünschenswert und könnte z. B. durch Verwendung standardisierter Datenblätter und Erhebung vorgegebener Parameter erfolgen.

Nachteilig an unserer retrospektiven Auswertung ist auch, dass die Erfassung der Entscheidungsbegründung zur Wahl des Transportmodus (luftgebunden vs. bodengebunden bzw. direkt vs. indirekt) fehlt, und somit nicht hinsichtlich ihrer Konsequenzen ausgewertet werden kann. Unklar ist auch, ob bereits im Rahmen der Erstversorgung „on the scene" die arterielle Verletzung vermutet oder diagnostiziert wurde.

Es stellt sich die Frage, ob die unsererseits erhobenen Daten auf andere Regionen übertragbar sind. Dies erscheint jedoch aufgrund der mit anderen Regionen nicht vergleichbaren alpinen Geografie im Bundesland Tirol problematisch. In anderen Regionen treten andere Verlet-

\section{Preclinical data on traumatic arterial limb injuries. Is the outcome influenced by transportation modality and distance?}

Background: The University Hospital of Innsbruck is a level I trauma center located in the Alps with a large catchment area. We report on selected aspects of preclinical care (transportation modality, direct vs. indirect and by helicopter vs. rescue van) in patients with traumatic arterial injuries to upper or lower limbs and their influence on outcome parameters (survival, limb preservation, follow-up operations and length of hospital stay).

Material and methods: All patients treated at the Department of Vascular Surgery between 2004 and 2018 after a trauma-related injury to an extremity artery were retrospectively identified and included. Exclusion criteria were iatrogenic injuries, lesions treated conservatively, by ligation or embolization and lesions to side branches of main arteries.

Results: In total, 113 patients were included (upper extremity: $n=59$, lower extremity: $n=56$ ). Trauma mechanisms were $34.5 \%$ sports, $23 \%$ work-related and $20.4 \%$ trafficrelated injuries. Patient transportation was more frequently direct than indirect $(59.3 \%$ vs. $40.7 \%, p<0.05)$ and differences were seen in upper vs. lower limbs related to injury mechanisms and transportation. Both direct and indirect transportation were significantly more frequent using air routes. The distance to the accident location and transportation modality were not correlated with poor outcome (survival, limb preservation, follow-up operations and length of hospital stay).

Conclusion: In our surroundings in an Alpine region, centralization of patient care in traumatic extremity artery injuries does not per se lead to worse results in patients with longer transportation distances, in patients transferred indirectly and in patients coming with rescue vans instead of helicopters. Whether our data are transferable to other regions should be the subject of future studies.

\section{Keywords}

Vascular injury · Extremity · Prehospital · Patient transportation · Outcome zungsmuster (z.B. keine typischen „Alpinunfälle" bzw. keine Sportverletzungen in schwer zugängigem Gelände) auf und bestehen andere Versorgungsstrukturen (keine abgelegenen Täler mit einer einzigen Zufahrtsstraße). Unter Umständen gibt es auch keine jahrzehntelang etablierte Zentralisierung der gefäßchirurgischen Versorgung. Unserer Daten belegen, dass eine Versorgung von Extremitätenarterienverletzungen zentralisiert erfolgen kann, also präferenziell in einem gefäßchirurgischen Zentrum der Region, auch wenn es vom Unfallort oftmals weiter entfernt ist als ein anderes Krankenhaus. Gleichzeitig bestätigt sie, dass die zügig durchgeführte Erstversorgung und Erstuntersuchung in einem Krankenhaus der Grundversorgung und eine anschließende Transferierung in ein gefäßchirurgisches Zentrum zu vergleichbaren Resultaten führt wie die direkte Einlieferung.

\section{Limitationen}

Unsere Datenauswertung ist durch typische Probleme einer retrospektiven Expost-Analyse limitiert. Für einzelne Parameter bestehen inkomplette Datensätze. Folgende Aspekte sind nicht bzw. nur mangelhaft dokumentiert: die Begründung der Entscheidung zum Transportmodus (luft- vs. bodengebunden bzw. direkt vs. indirekt); die "Zeitachse"; die Nichtberücksichtigung von Todesfällen vor der Krankenhauseinlieferung bzw. vor dem Behandlungsbeginn (z.B. auf dem Weg vom Schockraum in den OP).

\section{Fazit für die Praxis}

- Die Entfernung des Unfallorts und die Transportmodalität haben im Bundesland Tirol bei Extremitätenarterienverletzungen keinen signifikanten Einfluss auf die klinischen Ergebnisparameter und die Versorgungsqualität.

- Es gibt in der gefäßchirurgischen Literatur keine Belege dafür, bei Extremitätenarte- 
rienverletzungen aufgrund der klinischen Ergebnisse eine Transportmodalität oder die direkte Einlieferung zu bevorzugen. Die spätere Zutransferierung nach Erstversorgung führt zu vergleichbaren $\mathrm{Er}$ gebnissen.

- Die Daten aus Tirol sind nur eingeschränkt auf andere Regionen übertragbar, unterstützen aber eine zentralisierte Patientenversorgung von Extremitätenarterienverletzungen auch bei großem Einzugsgebiet.

\section{Korrespondenzadresse}

\section{PD Dr. med. Josef Klocker, MSc}

Universitätsklinik für Gefäßchirurgie, Medizinische Universität Innsbruck Anichstr. 35, 6020 Innsbruck, Österreich josef.klocker@i-med.ac.at

Funding. Open access funding provided by University of Innsbruck and Medical University of Innsbruck.

\section{Einhaltung ethischer Richtlinien}

Interessenkonflikt. J. Klocker, S.A.E. Heuberger, A. Gratl, M. Kluckner und S. Wipper geben an, dass kein Interessenkonflikt besteht.

Für diesen Beitrag wurden von den Autoren keine Studien an Menschen oder Tieren durchgeführt. Für die aufgeführten Studien gelten die jeweils dort angegebenen ethischen Richtlinien.

Open Access. Dieser Artikel wird unter der Creative Commons Namensnennung 4.0 International Lizenz veröffentlicht, welche die Nutzung, Vervielfältigung, Bearbeitung, Verbreitung und Wiedergabe in jeglichem Medium und Format erlaubt, sofern Sie den/die ursprünglichen Autor(en) und die Quelle ordnungsgemäß nennen, einen Link zur Creative Commons Lizenz beifügen und angeben, ob Änderungen vorgenommen wurden.

Die in diesem Artikel enthaltenen Bilder und sonstiges Drittmaterial unterliegen ebenfalls der genannten Creative Commons Lizenz, sofern sich aus der Abbildungslegende nichts anderes ergibt. Sofern das betreffende Material nicht unter der genannten Creative Commons Lizenz steht und die betreffende Handlung nicht nach gesetzlichen Vorschriften erlaubt ist, ist für die oben aufgeführten Weiterverwendungen des Materials die Einwilligung des jeweiligen Rechteinhabers einzuholen.

Weitere Details zur Lizenz entnehmen Sie bitte der Lizenzinformation auf http://creativecommons.org/ licenses/by/4.0/deed.de.

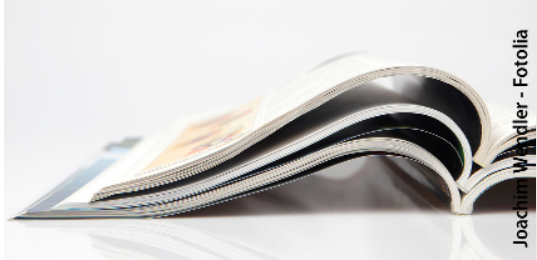

\section{Haben Sie anderes Papier bei Ihrer Zeitschrift be- merkt?}

Eine Mitteilung von Springer Nature / Springer Medizin

Wie viele andere Industriezweige hatte auch die Papierherstellung in den letzten Monaten mit Problemen in der Lieferkette zu kämpfen. Darüber hinaus stellten einige Papierfabriken von der Herstellung von Druckpapier auf die profitableren Verpackungsmaterialien um, insbesondere Karton wegen des boomenden OnlineHandels. Das verfügbare Papiervolumen ging Anfang dieses Jahres weiter zurück, da mehrere große Papierfabriken, darunter auch eine, auf die Springer Nature angewiesen ist, wegen eines Streiks geschlossen wurden.

\section{Papierknappheit wird anhalten}

Der Krieg in der Ukraine hat die ohnehin schon angespannte Lage auf dem globalen Papiermarkt, insbesondere in Europa, noch verschärft. Embargos für Holzexporte und Energielieferungen aus Russland werden die Papierknappheit weiter befeuern.

\section{Druckbetrieb bleibt unverändert}

In diesem Umfeld setzt Springer Nature seinen Druckbetrieb unverändert fort, ist aber mit Beschaffungsschwierigkeiten konfrontiert, die eine Flexibilität bei der Papiersorte für jede einzelne Zeitschrift erfordern. Wenn Sie also anderes Papier als bisher bei Ihrer Zeitschrift entdecken, so kennen Sie jetzt den Hintergrund. Springer Nature wird die gewohnten Sorten liefern, wenn der Papiermarkt dies zulässt. Wir bitten um Ihr Verständnis. 